$\begin{array}{cl}\begin{array}{c}\text { Revue } \\ \text { de } / \text { histoire }\end{array} & \text { Revue de l'histoire des religions } \\ \text { des religions } & 2 \mid 2009 \\ & \text { Varia }\end{array}$

L'histoire mouvementée d'Aimee S. McPherson (1890-1944), la première grande prédicatrice américaine du $\mathrm{xx}^{\mathrm{e}}$ siècle

The Agitated Story of Aimee S. McPherson (1890-1944): the First Greatest American Woman Preacher of the XX $X^{\text {th }}$ Century

Mokhtar Ben Barka

\title{
OpenEdition
}

Journals

Édition électronique

URL : http://journals.openedition.org/rhr/7234

DOI : $10.4000 /$ rhr.7234

ISSN : $2105-2573$

Éditeur

Armand Colin

Édition imprimée

Date de publication : 1 avril 2009

Pagination : 227-246

ISBN : 978-2200-92590-1

ISSN : 0035-1423

Référence électronique

Mokhtar Ben Barka, «L'histoire mouvementée d'Aimee S. McPherson (1890-1944), la première grande prédicatrice américaine du xx siècle », Revue de l'histoire des religions [En ligne], 2 | 2009, mis en ligne le 01 avril 2012, consulté le 30 avril 2019. URL : http://journals.openedition.org/rhr/7234; DOI :

$10.4000 /$ rhr.7234 


\section{L'histoire mouvementée d'Aimee S. McPherson (1890-1944), la première grande prédicatrice américaine du $\mathrm{xx}^{\mathrm{e}}$ siècle}

La biographie de la prédicatrice Aimee Semple McPherson révèle une personnalité originale, complexe et contradictoire. Elle était atypique dans la mesure où ses méthodes de prédication étaient jugées hors norme par les grandes Églises protestantes. En effet, elle faisait appel aux sentiments dans ses sermons et utilisait volontiers le déguisement et la mise en scène. "Sister 》 Aimee était aussi atypique, parce que sa vie était émaillée de scandales moraux et financiers. Par ses excentricités, sa démesure et sa maîtrise des techniques commerciales, elle augurait les pratiques, aussi bien que les dérives, des télévangélistes modernes, tels que Jerry Falwell, Jimmy Swaggart et James Bakker.

The Agitated Story of Aimee S. McPherson (1890-1944) : the First Greatest American Woman Preacher of the XXth Century

The biography of the preacher Aimee Semple McPherson unveils an unconventional, complex and contradictory personality. She was atypical insofar as her preaching methods were considered to be peculiar by mainline Protestant churches. In fact, in her sermons she appealed to feelings, enjoyed being in costume, and willingly resorted to production techniques. "Sister" Aimee was also atypical, because her life was punctuated by moral and financial scandals. By her eccentricities, her immoderation, and her command of marketing techniques, "Sister" Aimee foreshadowed the practices as well as the excesses of presentday televangelists, such as Jerry Falwell, Jimmy Swaggart and James Bakker. 
Aimee Semple McPherson, appelée plus couramment « Sister ${ }^{1}$ Aimee, était - pour reprendre une expression due à l'historien Nathan Hatch - « un phénomène typiquement américain »². De 1918 à sa mort en 1944, elle avait régné sur l'Angelus Temple, à Los Angeles, et subjugué des centaines de milliers de personnes à travers le pays. Figure marquante des années vingt, elle était aussi célèbre que les grandes vedettes hollywoodiennes de l'époque, Greta Garbo, Jean Harlow, ou Mary Pickford. Connue pour sa pratique de l'évangélisation itinérante ( barnstorming »), elle fut l'unique évangélisatrice capable de rivaliser avec la popularité des plus grands prédicateurs de l'époque : Dwight L. Moody, Charles Haddon Spurgeon et Billy Sunday. Sa notoriété s'étendait du Canada à la Floride et de la Nouvelle Angleterre à la Californie. «Aucun touriste, qui visitait la Californie dans les années vingt, note Daniel Mark Epstein, n'avait le sentiment que son voyage était réussi s'il ne pouvait se vanter d'avoir assisté à l'un des sermons de Aimee $»^{3}$.

Le parcours d'Aimee $\mathrm{S}$. McPherson révèle une personnalité hors norme, complexe et contradictoire. Personnage atypique en raison de l'originalité de son style de prédication, elle faisait appel dans ses sermons à l'intensité des sentiments et utilisait le déguisement et la mise en scène. Son penchant pour les excès spectaculaires était si fort que son message devenait souvent secondaire. Si elle dérangea nombre de ses contemporains, ce fut aussi parce que sa vie privée, particulièrement agitée, fut entachée de scandales moraux et financiers. On conviendra que pour une prédicatrice, ceci ne constitue pas le moindre des paradoxes.

Les excentricités d'Aimee $\mathrm{S}$. McPherson suscitèrent les réactions les plus contrastées, d'indignation comme d'admiration. Alors que ses frasques et ses pratiques, charlatanesques pour de nombreuses

1. L'usage des termes « sœur » et « frère » relève d'une habitude largement répandue dans l'ensemble des mouvements évangéliques, le pentecôtisme inclus. Il s'inscrit dans la logique d'un modèle congrégationnaliste en opposition à un modèle multitudiniste.

2. Nathan Hatch, "Preface", in Edith L. Blumhofer, Aimee Semple McPherson. Everybody's Sister, Grand Rapids, Michigan, William B. Eerdmans Publishing Company, 1993, p. ix.

3. Daniel Mark Epstein, Sister Aimee. The Life of Aimee Semple McPherson, New York, Harcourt Brace Jovanovich Publishers, 1993, p. 259. 
personnes, révulsaient les grandes Eglises traditionnelles (mainline churches), elle était admirée par un large public de gens, de condition modeste et sensibles à la mobilisation d'un registre émotionnel. L'histoire de cette prédicatrice, typique et étrange à la fois, s'inscrit dans les paradoxes qui ont marqué les " années folles ». Pour bien comprendre la complexité de sa personnalité, il importe de s'intéresser aux événements les plus marquants de son parcours, afin de démontrer dans quelle mesure « Sister » Aimee fut effectivement une prédicatrice hors norme. Enfin, l'exceptionnalisme des années vingt mérite une attention particulière, qui permettra de mieux comprendre ce personnage si singulier que fut Aimee S. McPherson.

\section{UN ÉTRANGE DESTIN}

« Sister » Aimee fut indiscutablement la première grande prédicatrice de l'histoire religieuse américaine. Il n'empêche que son nom reste associé dans la mémoire collective aux innombrables scandales qui jalonnèrent son parcours. Lorsqu'on examine attentivement sa biographie, on se rend compte que sa vie fut une succession d'événements plus étranges les uns que les autres, à commencer par les remous provoqués par ses parents bien avant sa naissance. On est ainsi tenté de penser qu'elle fut prédestinée à connaître un tel sort. En effet, sa mère, Mildred (dite Minnie) Pearce, orpheline dès l'âge de douze ans, fut employée par le futur père d'Aimee, James Kennedy, pour s'occuper de sa première femme, Elizabeth, alors gravement malade. Lorsque celle-ci décéda, quelques mois plus tard, James Kennedy, bien qu'âgé alors de cinquante ans, décida d'épouser la jeune Minnie, qui n'en avait que quinze. La différence d'âge entre les futurs époux déclencha un tel tollé à Salford, petit village de l'Ontario, qu'ils durent partir dans le Michigan pour pouvoir se marier.

Née en 1890, Aimee Elizabeth Kennedy grandit dans une famille où la religion occupait une place prépondérante. Minnie Kennedy, qui avait rejoint l'Armée du Salut à l'âge de douze ans ${ }^{4}$, baptisa sa

4. L'Armée du Salut est un mouvement religieux fondé en Angleterre en 1865 , en pleine révolution industrielle, par le pasteur méthodiste William Booth et son épouse Catherine, saisis par le drame de la condition ouvrière. Aujourd'hui, l'Armée du Salut est une structure internationale implantée dans une centaine de pays. 
fille dans la tradition salutiste, alors qu'elle n'avait que six mois ${ }^{5}$. Elle s'efforça également de l'élever dans la foi chrétienne et de lui donner une solide culture biblique. Au lieu des contes de fées et des comptines traditionnelles, Minnie lui racontait des histoires bibliques et lui apprenait hymnes et cantiques. Il en alla de même pour son père, un méthodiste fervent, qui tenait à ce que sa fille ait une bonne éducation religieuse. Comme le fait remarquer Edith L. Blumhofer, « les deux parents prodiguèrent de l'amour à leur fille unique et s'assurèrent qu'elle assimilait des connaissances bibliques approfondies ». Le résultat fut que « à cinq ans, elle connaissait des pans entiers de l'Écriture sainte et était capable de raconter, à la demande, n'importe quelle histoire biblique $»^{6}$. Aussi participaitelle assidûment aux activités de l'Eglise méthodiste de son village. La jeune Aimee se distingua très tôt, en outre, par son talent d'oratrice : « à l'âge de treize ans, Aimee était une oratrice publique réputée $»^{7}$. Audacieuse et dotée d'une voix tonitruante, elle savait capter l'attention de son auditoire. C'est pourquoi elle était souvent invitée à animer les fêtes scolaires ainsi que les spectacles organisés par son Eglise.

Dès son jeune âge - et en dépit de sa solide éducation religieuse -, Aimee s'était passionnée pour le théâtre, la musique et la danse, des loisirs jadis considérés comme impies par certaines mouvances évangéliques conservatrices. Plus encore, elle connut dans son adolescence des crises existentielles. Ainsi, elle en vint à remettre en cause la foi et les croyances inculquées par ses parents, et même à douter de l'existence de Dieu. Sa quête de sens l'amena à lire des auteurs comme Voltaire, Ingersoll, Paine et Darwin et à engager des discussions avec les pasteurs de son village. Elle adressa même une lettre ouverte au plus grand journal de Montréal, le Family Herald and Weekly Star, dans laquelle elle soutenait d'une manière à peine voilée la thèse darwinienne de l'évolution, provoquant la consternation non seulement de ses parents, mais aussi des habitants de son village.

Mais son scepticisme et sa quête de vérité furent de courte durée, et à l'âge de seize ans, elle retrouva la foi et se convertit au

5. Silvia Anne Sheafer, Aimee Semple McPherson, Philadelphia, Chelsea House Publishers, 2004, p. 13.

6. Edith L. Blumhofer, op. cit., p. 13.

7. Daniel Mark Epstein, op. cit., p. 28. 
pentecôtisme, une religion dotée, à l'époque, d'une très mauvaise réputation, encore confortée par l'attirance exercée sur les couches populaires. Honnis étaient ceux - et surtout celles - qui acceptaient de faire l'expérience du «baptême du saint Esprit » ${ }^{8}$, l'un des piliers de la foi pentecôtiste qui se traduit généralement par le « parler en langues $»^{9}$ et par les « guérisons miraculeuses » (appelées aussi «divine healing »). Aimee eut, de fait, un coup de foudre pour un prédicateur pentecôtiste itinérant, Robert Semple, qu'elle épousa en août 1908, peu après leur première rencontre dans l'une des Eglises du village ${ }^{10}$. Lors de la célébration du culte à laquelle elle participait pour la première fois en compagnie de son père, Aimee fut impressionnée par l'abattage du prédicateur Semple, et par la puissance de son sermon, qui fut un tournant dans sa vie: « Dès cet instant, je n'ai jamais douté que c'était Dieu qui s'était manifesté à moi... pour me révéler ma véritable condition ... de pécheresse égarée, misérable et digne de l'enfer $»^{11}$. Mais, «il est juste de dire, affirme Daniel Mark Epstein, que la conversion d'Aimee était due à la religion pour un dixième et à des sentiments d'amour pour le reste ». Et le même auteur d'ajouter : « Son ministère allait être à tout jamais imprégné de l'esprit d'Eros $»^{12}$. Minnie Kennedy se montra déçue parce qu'elle aurait aimé voir sa fille servir dans les rangs de l'Armée du Salut, et outrée du fait que Robert

8. Pour les pentecôtistes, le «baptême du saint Esprit» est une deuxième expérience après « la nouvelle naissance » qui résulte de la conversion. Ils considèrent que celui qui a la foi a reçu l'Esprit Saint, mais qu'il est nécessaire par la suite de faire le « baptême du saint Esprit ».

9. Dans les milieux pentecôtistes et charismatiques, le " parler en langues" (appelé aussi " glossolalie ») est un mode d'expression public par lequel le locuteur s'exprime avec des sons censés venir d'une langue inconnue. Ce mode d'expression qui marque le baptême du Saint-Esprit, devient alors une preuve quasi quotidienne qui constitue un véritable marqueur de la foi pentecôtiste, une preuve de l'authenticité de la conversion. La source du parler en langues se trouve dans l'expérience de la Pentecôte aux débuts de l'Eglise (voir Actes 2, $1-13)$.

10. La doctrine commune à la majorité des pentecôtistes fut formulée pour la première fois, en 1901, par Charles Fox Perham, qui dirigeait une école biblique à Topeka dans le Kansas. Mais ce fut avec l'évangéliste noir William James Seymour que le pentecôtisme prit naissance en 1906. Avant de s'imposer en tant que mouvement indépendant, le pentecôtisme s'exprimait comme un mouvement de renouveau à l'intérieur des Eglises évangéliques.

11. Aimee Semple McPherson, This is That, Los Angeles, Foursquare Publications, 1923, p. 37.

12. Daniel Mark Epstein, op. cit., p. 47. 
Semple fût pentecôtiste et, qui plus est, sans ressources. La réaction de James Kennedy fut identique : " Le père d'Aimee, pour sa part, était déconcerté. Ils [les kennedy] étaient chrétiens mais ne comprenaient pas le parler en langues ni la doctrine religieuse ultraconservatrice des pentecôtistes $\|^{13}$.

$\mathrm{Au}$ lendemain de leur mariage, Robert Semple fit part à sa jeune épouse de son ardent désir de partir en Chine en tant que missionnaire : « La Chine forte de ses millions d'habitants, lui dit-il, constituait un défi constant pour le christianisme. Et là - cette toute petite île de Macao - c'est ma destination et le point de départ de mon offensive $»^{14}$. Aimee ne pouvait que partager l'enthousiasme de son mari, d'autant qu'elle ne cessait, disait-elle, d'entendre en elle l'appel du Seigneur à « prêcher la Bonne Parole ». En juin 1910, les Semple débarquèrent à Hong Kong. Mais, peu de temps après leur arrivée, Robert Semple décéda de dysenterie, et un mois plus tard, Aimee donna naissance à une fille, Roberta Star. De retour de Chine, juste après la naissance de sa fille, Aimee débarqua à New York, où Minnie Kennedy, ayant abandonné son mari, s'était installée pour se consacrer à l'Armée du Salut. En février 1912, Aimee fit la connaissance d'Harold $\mathrm{McPherson}^{15}$, un jeune comptable originaire de la Rhode Island, qu'elle épousa en secondes noces. De leur union naquît, l'année suivante, un garçon prénommé Rolf. Non qu'Aimee fût amoureuse de McPherson, mais elle accepta de l'épouser essentiellement pour offrir un toit et un cadre familial à sa fille Roberta Star. Aimee n'avait rien d'une femme au foyer, au grand dam de son époux. Se sentant investie d'une mission divine, elle était résolue à partir à " la conquête des âmes pour le Seigneur », en organisant des « réveils » religieux partout où cela serait possible. « Depuis que j'étais toute petite, écrit Aimee, j'ai senti - et je sais maintenant que ce que j'ai senti durant tout ce temps était la promesse de Dieu, qu'Il m'appellerait pour Le servir et Lui gagner des âmes $»^{16}$.

13. Silvia Anne Sheafer, op. cit., p. 22.

14. Aimee Semple McPherson, The Story of My Life, Waco, World Books, 1951, p. 33.

15. Harold fit l'expérience de la conversion en 1907 lors d'un rassemblement revivaliste animé par l'évangéliste Gipsy Smith à Providence dans la Rhode Island. Il s'apprêta même à devenir ministre du culte, mais pour des raisons familiales il dut y renoncer. Voir Edith L. Blumhofer, op. cit., p. 102.

16. Aimee Semple McPherson citée in Robert Bahr, Least of All Saints, Englewood Cliffs, Prentice Hall, 1979, p. 35. 
Déterminée à continuer l'action missionnaire de son premier mari, elle entreprit, en 1915, de parcourir les Etats-Unis à bord d'une vieille voiture baptisée "Gospel Car ${ }^{17}$, accompagnée de sa mère, de ses deux enfants (Roberta et Rolf) et d'un petit groupe d'assistants. Quant à Harold McPherson, il accepta, au début, de l'accompagner dans son ministère itinérant. Mais il se fatigua très rapidement de la vie de bohème choisie par son épouse. Déçue par sa lassitude, Aimee n'hésita pas à s'en séparer. La rupture n'affecta nullement Aimee, «pas plus d'ailleurs que McPherson, lequel ne tenta jamais de reconquérir sa capricieuse moitié $»^{18}$.

Debout dans sa voiture, un mégaphone à la main, Aimee haranguait la foule, en répétant inlassablement que le retour du Christ était imminent. Conjointement à l'appel à la conversion personnelle, elle pratiquait les "guérisons miraculeuses ", si bien que les malades, les non-voyants, les malentendants et les paralysés allaient à sa rencontre, par centaines de milliers, partout où elle se trouvait. Et en dépit des attaques de ses détracteurs, elle fit souvent merveille; les conversions et les " guérisons » furent légion. "Partout où elle allait, écrit Daniel Mark Epstein, elle créait l'événement et les gens s'en souvenaient et en parlaient $»{ }^{19}$. Cela contribua sans doute à sa fulgurante ascension, largement commentée par les plus grands quotidiens américains, tels que le Los Angeles Times et le New York Times. Mais voilà, Aimee connut, dans le même temps, de sérieux problèmes de santé. Malgré les apparences, elle était non seulement fragile ${ }^{20}$, mais également sujette à la dépression - le comble du paradoxe pour une " guérisseuse ».

Au mois d'octobre 1918, Aimee et son petit groupe arrivèrent à Los Angeles. Aussitôt, elle commença à prêcher un peu partout dans la ville. "Son succès fut tel, précise Jacques Chouleur, que ses admirateurs se cotisèrent pour lui offrir en pleine propriété une

17. Il s'agissait à l'origine d'une vieille Packard (modèle de 1912), remplacée par une Oldsmobile toute neuve. Sur un côté de la « Gospel Car », il y avait cette inscription : «JESUS IS COMING SOON - GET READY! ». Sur l'autre côté, on pouvait lire : « WHERE WILL YOU SPEND ETERNITY? ».

18. Jacques Chouleur, «Le panorama religieux de l'Amérique des années 1920 », in Serge Ricard, The Twenties, Aix-en-Provence, Publications de l'Université de Provence, 1982, p. 114.

19. Daniel Mark Epstein, op. cit., p. 117.

20. Elle subit deux opérations chirurgicales en 1917. 
belle maison d'habitation $»^{21}$, que «Sister » Aimee baptisa «The House That God Built ». A cet égard, il est légitime de penser que le choix de la prédicatrice et de sa famille de s'installer à Los Angeles n'était pas dû au hasard: la Californie, connue naguère comme aujourd'hui pour sa tolérance et son esprit de liberté, a toujours été la terre d'élection des excentriques en tout genre.

A peine installée dans sa nouvelle maison, «Sister » Aimee entama une grande tournée d'évangélisation à travers les EtatsUnis et le Canada. Grâce aux dons qui affluaient de partout, elle inaugura, en 1923, son temple personnel, Angelus Temple, que Jacques Chouleur décrit comme « un énorme édifice circulaire surmonté d'un dôme de béton incrusté de coquillages marins, ... [dont] l'intérieur était conçu davantage comme un théâtre que comme un lieu de prière, avec une scène où officiait "Sister" Aimee, et une décoration somptueuse, mais d'assez mauvais goût, un peu dans le style hollywoodien d'alors $»^{22}$. A l'intérieur, il y avait un auditorium de 5300 sièges et un grand nombre de salles et de services annexes, y compris une station de radio (Kall Four Square Gospel - KFSG). Sans oublier l'orchestre composé de quatorze musiciens et d'une cinquantaine de choristes. En 1925, « Sister » Aimee fonda une école biblique, baptisée Lighthouse of International Fourquare Evangelism (LIFE) Bible College, dont les bâtiments jouxtaient l'Angelus Temple. L'on notera que ce temple, dont l'inauguration avait fait la une de la presse nationale et régionale, permit à « Sister » Aimee de réaliser son rêve, puisqu'en 1927, elle créa sa propre « dénomination» religieuse, l'International Church of the Foursquare Gospel.

« Sister» Aimee était l'objet d'une attention particulière de la part des journalistes mais aussi des hommes de loi, car elle dut engager ou subir d'innombrables procès de toute sorte. Ses relations avec les médias furent des plus ambivalentes : elle fut adulée autant que critiquée, comme en témoigne le scandale provoqué par son prétendu « kidnapping » que les journalistes exploitèrent sans vergogne. Le 18 mai 1926, l'évangélisatrice disparut alors qu'elle se baignait à Ocean Park, et ce, bien qu'elle fût excellente nageuse. Les recherches menées à l'aide de diverses embarcations s'avé-

21. Jacques Chouleur, op. cit., p. 114.

22. Ibid. 
rèrent infructueuses. Tout le monde la crut noyée. Soudain, après cinq semaines d'absence, Aimee reparut, affirmant qu'elle avait été enlevée par deux hommes et une femme, qui l'avaient emmenée au Mexique, où elle avait été emprisonnée et brutalisée. Mais, elle avait réussi, dit-elle, à défaire ses liens et à s'enfuir dans le désert. On l'avait retrouvée en Arizona. La police ne crut pas, un seul instant, à la thèse de l'enlèvement. Et pour cause : ni la robe ni les souliers de la « victime » ne portaient la moindre trace de sa fuite à travers le désert. En revanche, plusieurs témoignages permirent de conclure qu'il s'agissait d'une escapade préméditée : Aimee S. McPherson avait voulu s'offrir une discrète aventure avec le technicien de sa station de radio, Kenneth Ormiston, marié et père d'un petit garçon. L'absence inexpliquée d'Ormiston du domicile conjugal pendant l'épisode de l' « enlèvement » ainsi que le témoignage de plusieurs personnes qui avaient affirmé l'avoir vu en compagnie d'une dame louer un chalet pour vacanciers, « donnèrent une certaine crédibilité à l'hypothèse [de l'escapade], en dépit des dénégations indignées d'Aimee et du silence impénétrable d'Ormiston $»^{23}$. Ce scandale, qui donna lieu à des poursuites judiciaires, faillit coûter très cher à Aimee ; le non-lieu qu'elle obtint de justesse la sauva, sans aucun doute, de la catastrophe, Son image n'en resta pas moins ternie aux yeux de beaucoup de personnes : "A Hollywood aucune vedette de cinéma ne brilla avec autant d'éclat que Aimee S. McPherson [...] mais à présent, Sœur Aimee était considérée comme un embarras pour toute la ville et un spectacle vulgaire $»^{24}$. A ce jour, les circonstances de son « enlèvement » demeurent inexpliquées.

A Angelus Temple l'ambiance était des plus détestables. « Sister » Aimee était entourée de conseillers et d'assistants qui se livraient à toutes sortes de manigances, avec pour conséquence l'éviction de Minnie Kennedy - chargée de la gestion des affaires de sa fille -, car jugée encombrante. Qui plus est, au cours d'une dispute survenue juste avant que Minnie ne quittât le Temple, Aimee frappa sa mère au visage et lui cassa le nez. Livrée à elle-même, la prédicatrice commit plusieurs erreurs financières, donnant lieu à de nombreux procès.

Malade et se sentant de plus en plus seule, «Sister » Aimee décida, en 1931, de se marier pour la troisième fois, avec David

23. Ibid., p. 117.

24. Daniel Mark Epstein, op. cit., p. 300. 
Hutton, un chanteur de sa chorale. Comme son second mariage, le troisième se solda par un divorce, au bout de trois ans de vie commune. A la même période, son état de santé se détériora, en partie à cause du surmenage : elle prêchait une vingtaine de fois par semaine, animait les émissions radiophoniques, éditait un magazine (Bridal Call) et composait sketches et opérettes. Encore plus préjudiciables à sa santé furent les querelles l'opposant à sa fille Roberta Star qui finit par lui faire ses adieux définitifs, en 1936.

En octobre 1944, Aimee S. McPherson mourut à l'âge de cinquante trois ans d'une surdose de somnifères. Bien que certains aient pensé qu'il s'agissait d'un accident, la thèse du suicide n'a jamais été totalement écartée. Comme le fait remarquer justement Edith L. Blumhofer, « la mort soudaine de Sœur Aimee à Oakland en octobre 1944, tout comme une bonne partie de sa vie, suscita des questions dérangeantes $»^{25}$. Il faut enfin dire que les funérailles d'Aimee McPherson étaient hors du commun : un cortège de 600 voitures porta sa dépouille au Forest Lawn Memorial Park Cimetery, situé à Glendale en Californie ${ }^{26}$.

La biographie de «Sœur» Aimee dévoile une existence mouvementée, ponctuée de plusieurs scandales, qui se termina d'une manière plutôt mystérieuse. De sa soudaine conversion au pentecôtisme - l'objet de critiques virulentes - à sa mort suspecte, en passant par son présumé enlèvement, sa vie fut, à l'évidence, marquée du sceau de l'étrangeté. Si certaines de ses excentricités étaient le fait du destin, d'autres, au contraire, étaient dues à un choix délibéré fondé sur des convictions personnelles. Ainsi, elle n'hésita pas à braver les traditions et, par là-même, à se faire remarquer, notamment en montant en chaire ou encore en créant un style de prédication axé sur le spectaculaire.

\section{UNE PRÉDICATRICE HORS NORME}

Aimee S. McPherson fut une prédicatrice hors norme pour plusieurs raisons. D'abord, parce qu'elle fut la première femme amé-

25. Edith L. Blumhofer, op. cit., p. 8.

26. Thomas Lately, Storming Heaven. The Lives and Turmoils of Minnie Kennedy and Aimee Semple McPherson, New York, William Morrow and Company, Inc., 1970, p. 344. 
ricaine à déclarer ouvertement sa vocation de prêcher l'Evangile, une activité généralement réservée aux hommes. A l'exception des célèbres évangélisatrices anglaises, Evangeline et Catherine Booth, les prédicatrices étaient rares à cette époque, que ce soit aux EtatsUnis ou dans le reste du monde chrétien. Nul étonnement, dès lors, qu'elle fût critiquée par d'autres ministres du culte : «Quand Aimee Semple McPherson commença vraiment à prêcher, une femme qui montait en chaire n'était pas seulement une nouveauté, mais la cible de critiques de la part des autres ministres du culte $»^{27}$. Rappelons que l'exercice de pareille activité avait toujours été refusé aux femmes, au motif que toute velléité de leur part romprait avec la tradition chrétienne, et plus spécifiquement avec les enseignements de l'Apôtre Paul qui, dès le premier siècle, avait vigoureusement affirmé que les femmes ne pouvaient prêcher (1ère Epître aux Corinthiens, $14: 34-36$; 1ère Epître à Timothée $2: 12-15$ ). Mais Aimee S. McPherson estimait, pour sa part, que la femme avait le même droit d'évangéliser que l'homme : « Une femme doit prêcher conformément aux Écritures si elle estime que cela relève véritablement de son devoir $»^{28}$.

Ensuite, Aimee S. McPherson devint dès 1922 la première femme à prêcher à la radio, puis la première à obtenir une licence pour émettre sur les ondes. Aidée par sa mère et par l'ingénieur du son Kenneth Ormiston, elle créa en février 1924 sa propre station de radio Kall Foursquare Gospel (KFSG). Tous les matins, elle animait en direct une émission baptisée "The Sunshine Hour ». Au bout de deux ans, KFSG arrêta cependant d'émettre, la commission fédérale chargée des communications (Federal Communications Commission - FCC) ayant refusé de renouveler sa licence. Il importe de savoir que la première station de radio à vocation commerciale, KDKA, établie à Pittsburgh par Westinghouse Electric, avait inauguré ses émissions le 2 novembre 1920. Deux mois jour pour jour plus tard, le 2 janvier 1921, KDKA diffusait la première émission religieuse : le culte dominical de la Calvary Episcopal Church à Pittsburgh. C'est dire combien « Sister» Aimee fut pionnière en la matière.

27. Silvia Anne Sheafer, op. cit., p. 37.

28. Aimee Semple McPherson citée par E. L. Blumhofer, op. cit., p. 195. 
En outre, «Sister» Aimee était atypique en raison de l'originalité de sa prédication, axée sur l'intensité émotionnelle et sur le culte de la personnalité. En l'écoutant, c'est l'émotion qui l'emportait sur la raison. Comme l'écrit Jacques Chouleur, « elle était belle, élégante, éloquente, souriante, émouvante, et ses dons de séduction infinis, à la fois sur les individus et sur les foules $»^{29}$. Elle était sympathique et son charme séduisait ses auditeurs comme ses interlocuteurs. Dans ces conditions, on comprend que le message religieux ait souvent été relégué au second plan.

Sur le plan doctrinal, son message, foncièrement pentecôtiste, se fondait sur les quatre croyances en la Régénération, la Guérison par la foi, le Baptême du Saint-Esprit et la seconde venue du Christ. Cela correspond au Foursquare Gospel: Jésus régénère, guérit, baptise et revient. Les prêches de « Sister » Aimee étaient rythmés d'extases mystiques, de baptêmes et de guérisons par imposition des mains. Bien que souvent comparée à Billy Sunday, connu pour les effets théâtraux auxquels il avait recours, elle s'en distinguait par un message plus optimiste et plus joyeux. Au lieu du châtiment de Dieu et de l'Enfer, thèmes favoris de la plupart des prédicateurs, y compris Billy Sunday, Aimee S. McPherson mettait en avant l'amour de Dieu et le pardon : «C'était une explosion ininterrompue de joie sans mélange. A Angelus Temple, il n'y avait ni mélancolie, ni bancs pour personnes en deuil, ni gémissements de pécheurs, ni sombre insistance sur la damnation et le feu de l'Enfer $»^{30}$. En écoutant « Sister » Aimee prêcher, chacun se sentait aimé personnellement. L'insistance sur le Dieu d'amour lui permettait de transcender les barrières " dénominationnelles » et donc de ratisser large. «Sister» Aimee avait cette autre particularité de mettre le salut à la portée de tout un chacun : «Elle proposait à chacun l'accès à une relation privilégiée et familiale avec Dieu, dont elle prétendait bénéficier, elle aussi $\|^{31}$. Ce faisant, elle contribua sans aucun doute à la « démocratisation » de l'expérience religieuse aux Etats-Unis.

Aimee S. McPherson possédait d'indéniables talents d'actrice. Son fort penchant pour les excès spectaculaires lui faisait rechercher les effets nouveaux ; elle adorait les déguisements et les uniformes.

29. Jacques Chouleur, op. cit., 114.

30. Thomas Lately, op. cit., p. 26.

31. Edith L. Blumhofer, op. cit., p. 20. 
Pour séduire son public, elle apparaissait sur scène en infirmière, en motard de la police, en capitaine des pompiers, ou encore en femme du Mayflower. Grâce à ses talents d'actrice, elle transformait l'Evangile en un spectacle hollywoodien, où se mêlaient le théâtre et la musique, et notamment le jazz. La proximité d'Hollywood lui permit de s'inspirer de certaines techniques cinématographiques et d'avoir accès aux accessoires dont elle avait besoin. Connus sous l'appellation « sermons illustrés» (illustrated sermons), ses prêches prirent souvent la forme de sketches nécessitant des décors, des costumes et de la musique. Elle transformait ainsi l'Eglise en un lieu de divertissement : "Ce fut ce mélange de piété et d'apparat qui fit de Sister une femme à sensation $»^{32}$. Outre son goût prononcé pour le spectacle et les déguisements, elle utilisait volontiers la langue de tous les jours, afin d'être comprise de tout le monde. Ce côté populiste joua certainement un rôle important dans le renforcement et la pérennité de sa popularité.

Aimee S. McPherson n'en était pas moins, aux yeux de beaucoup de gens, un personnage douteux. Le célèbre critique Henry L. Mencken la qualifia d'ailleurs de «charlatan manifestement ordinaire $»^{33}$. Il y avait chez elle, confirme Jacques Chouleur, « un charlatanisme qui s'alliait fort bien d'une paradoxale sincérité, du moins sur scène, dans le feu de la prédication $»^{34}$. Elle avait souvent recours à des artifices de mise en scène, comme le procédé consistant à remercier en chaire les admirateurs anonymes qui lui avaient envoyé un bouquet de fleurs qu'elle tenait à la main, et qu'elle faisait en réalité acheter chez le fleuriste par un assistant avant chaque prêche $^{35}$.

Particulièrement douée pour la collecte des fonds, Aimee S. McPherson savait faire appel à la générosité du public ; pour preuve les sommes considérables que lui rapportait la quête. Grâce aux dons de ses fidèles, elle s'était trouvée, en peu de temps, à la tête d'un « empire » de plusieurs centaines de millions de dollars. Pour satisfaire des besoins personnels, souvent excessifs, elle puisait immodérément dans la caisse, au mépris des recommandations de

32. Ibid., p. 231.

33. Henry L. Mencken cité in Charles Fecher, Mencken, New York, Alfred A. Knopf, 1978.

34. Jacques Chouleur, op. cit., p. 115.

35. Ibid. 
sa mère qui voulait une comptabilité honnête et transparente. Outre des croisières en Europe, aux Caraïbes, en Terre Sainte, elle s'offrit un manoir de style pseudo mauresque, situé au lac Elsinore, à une centaine de kilomètres de Los Angeles.

Le mélange des genres effectué par «Sister» Aimee lui valut d'être critiquée par les grandes Eglises de l'Establishment protestant, qui dénoncèrent l'appauvrissement et la «banalisation»du message religieux dus à l'exploitation des émotions et au recours au spectaculaire. Au lieu de servir l'Evangile, Aimee ne se souciait, selon ses détracteurs, que de s'attirer les dons du public. Les Eglises situées à proximité de l'Angelus Temple, qui voyaient diminuer le nombre de leurs fidèles, se joignirent aux critiques. Quant aux « dénominations » les plus conservatrices, elles s'accordaient à regretter que la principale vocation d'Aimee - comme celle de toutes les femmes - ne fût point de rester au foyer, conformément aux enseignements de la Bible.

Mais « Sister» Aimee n'avait cure des contradictions. Imprégnée de l'exemple maternel, elle délaissa très vite son second mari McPherson, qui ne partageait guère son enthousiasme religieux, alors qu'elle se disait opposée au divorce. Quant à son troisième mariage, en 1931, avec David Hutton, un coureur de jupons et grand buveur avéré, il contrevenait à l'une des doctrines établies par sa propre Eglise, qui interdisait le remariage tant que l'ex-conjoint était encore en vie. Etait-elle consciente de l'ampleur de son acte ? Ce qu'elle cherchait en contractant ce mariage était, expliqua-t-elle, «la protection d'un homme, le sérieux, la tendresse et le dévouement d'un bon époux $»^{36}$. En tout état de cause, de nombreuses Eglises quittèrent l'International Church of the Fourquare Gospel en signe de protestation.

Cependant, il ne faut pas oublier que la personnalité de « Sister » Aimee présentait aussi des facettes séduisantes, notamment la générosité et le patriotisme. Pendant la Grande Dépression consécutive au Krach boursier d'octobre 1929, Aimee mit en place, par exemple, l'Angelus Temple's Foursquare Commissary, dont la

36. Aimee Semple McPherson, The Story of My Life, p. 234. Avant de rencontrer Aimee S. McPherson, David Hutton avait eu une fiancée, Hazel St Pierre, à qui il aurait promis le mariage. Pour ne pas avoir tenu sa promesse, cette dernière l'avait poursuivi en justice et obtenu 5000 dollars de dommages et intérêts. Il fut également impliqué dans une affaire d'extorsion de fonds. 
mission était de venir en aide aux nécessiteux et autres victimes de la crise. Soupes populaires, soins médicaux, aides financières : la prédicatrice ne lésina sur aucun moyen. En l'espace d'un mois, l'Angelus Temple's Foursquare Commissary servit pas moins de 30000 repas.

La personnalité très controversée d'Aimee S. McPherson ainsi que son style de prédication, très original, lui valurent d'être qualifiée de charlatan. Il n'en reste pas moins vrai qu'elle était le pur produit de son époque. Ses excentricités avaient ses origines dans les mœurs et les mentalités des «années folles ». Bien plus, elle était à l'avant-garde de ce que l'on nomme aujourd'hui le « télévangélisme».

\section{UNE PERSONNALITÉ TYPIQUE DE SON ÉPOQUE}

Prise isolément, la personnalité d'Aimee S. McPherson était, à n'en point douter, complexe et étrange. Mais si on la replace dans le contexte des années vingt, on se rend compte que son originalité était intimement liée aux contradictions caractérisant cette période. Ainsi que le rappelle André Kaspi, «rien n'est plus divers que ce pays ni plus contradictoire que cette période de son histoire $»^{37}$. Au fond, Aimee était une femme de son temps ; sa personnalité et son parcours s'enracinaient dans une société transformée par des changements politiques, économiques, sociaux et culturels qui firent brusquement des Etats-Unis d'alors tout à la fois un modèle et un repoussoir, surprenant les Américains mais, quelquefois aussi, les désorientant.

Ce qui est remarquable à cet égard, c'est que l'étrangeté de «Sister» Aimee était bienvenue, voire réclamée par ses ouailles. Elle répondait, en quelque sorte, à un besoin d'extases émotionnelles éprouvé par un pan entier de la population américaine. Son public l'adorait car il estimait qu'elle était « dans la norme». En ce sens, Edith L. Blumhofer affirme que " malgré la fascination des gens pour ce que sa vie avait de théâtral et de sensationnel, Sœur Aimee était populaire en grande partie parce qu'elle était typique $»^{38}$. Aimee

37. André Kaspi, Les Etats-Unis au temps de la prospérité : 1919-1929, Hachette, 1994, p. 12.

38. Edith L. Blumhofer, op. cit., p. 18. 
S. McPherson était adulée par l'Amérique profonde, les habitants des petites villes du Middle West et du Sud, les fermiers, les classes moyennes, les laissés-pour-compte, les paumés, les malades, les personnes psychologiquement instables, tous à la recherche de sensations fortes et d'émotions intenses. L'admiration qu'avaient ces gens pour elle s'expliquait aussi par le fait qu'elle incarnait, à leurs yeux du moins, le rêve américain : beaucoup s'identifiaient à elle en raison de sa réussite et de sa notoriété. Comme bon nombre de ses admirateurs, elle avait des origines humbles. Issue d'un milieu modeste et sans aucune formation théologique, cette self-made woman devint une icône religieuse. Héroïne du rêve américain, son histoire ressemblait à une saga inscrite dans la tradition américaine. Qu'importe si les moyens utilisés pour réussir étaient discutables, seul le résultat comptait, estimaient ses défenseurs.

Evangélisatrice et femme émancipée à la fois, elle incarnait un mélange explosif de religion et d'érotisme, de tradition et de modernité. Ce serait imprudent, voire erroné, de la qualifier de féministe ou de "flapper », mais il est certain qu'elle tenta de se rapprocher de l'idéal féminin de l'époque. Elle avait beaucoup en commun avec la «femme nouvelle» ("new woman »), dans l'affirmation de sa personnalité et de son désir d'émancipation. Il ne fait aucun doute que la révolution dans l'habillement et le comportement caractéristique des « années folles » eurent un effet sur elle - « Sœur Aimee McPherson incarne l'esprit et l'image de la "nouvelle femme", bien que sa moralité réchaufferait le cœur du vieux Cotton Matther $»^{39}$. Comme toutes les jeunes femmes modernes, elle avait les cheveux coupés, portait chapeau-cloche, turban et bonnet - à la dernière mode -, et parcourut des milliers de kilomètres dans sa «Gospel car ». Toujours coquette, elle aimait se faire photographier avec un bouquet de fleurs à la main. Ses toilettes étaient innombrables et coûteuses, et elle fréquentait assidûment les instituts de beauté (et plus tard, dit-on, les chirurgiens esthétiques...). Comme le confirme Edith. L. Blumhofer : "Ses longs cheveux auburn étaient coupés courts et coiffés en boucles serrées et ondulées. Elle mettait du maquillage et portait des vêtements élégants, y compris des tailleurs sur mesure et des chapeaux chic $»^{40}$.

39. Daniel Mark Epstein, op. cit., p. 158.

40. Edith L. Blumhofer, op. cit., pp. 73-74. 
De même que le comportement et la personnalité d'Aimee $\mathrm{S}$. McPherson étaient, à maints égards, en conformité avec les mœurs et croyances de l'époque, ses excentricités étaient alors presque classiques et sans surprise. A Los Angeles, par exemple, les religions charlatanesques prospéraient bien avant qu'Aimee et sa famille s'y installèrent, et la quête du bonheur - à tout prix et tout de suite y était érigée en loi absolue. Voici comment l'essayiste Henry L. Mencken décrivait Los Angeles au début des années vingt : « Les ostéopathes, les chiropracteurs, et autres charlatans du même genre l'avaient depuis longtemps marquée en s'y installant. Elle grouillait de pandits, de spiritualistes, de scientistes chrétiens, de voyants et de leurs associés, les nécromanciens. Elle offrait des affaires juteuses aux spéculateurs immobiliers, aux courtiers en valeurs pétrolières, aux spécialistes des écoutes téléphoniques et ainsi de suite $»^{41}$.

\section{Conclusion}

Pour excentrique et novatrice qu'elle fût, Aimee Semple McPherson s'inscrit néanmoins dans la lignée des grandes figures des « Réveils » qui ont révolutionné les techniques d'évangélisation aux Etats-Unis au vingtième siècle. En rupture avec le style puritain, elle mit l'accent sur le spectaculaire, à la manière de Dwight Moody. De ce point de vue, elle rejoint les précurseurs du télévangélisme moderne. Par ses scandales, ses excentricités, son insistance sur le bien-être et le bonheur immédiats, son sens de la démesure et sa maîtrise des techniques du marketing, "Sister » Aimee augurait les pratiques, tout autant que les dérives, des télévangélistes modernes, tels que Jerry Falwell, Jimmy Swaggart et James Bakker ${ }^{42}$.

Après la disparition de «Sister» Aimee, son fils Rolf K. McPherson lui succéda à la tête de l'international Church of the Foursquare Gospel. Au bout de quarante-quatre ans, il fut remplacé successivement par Harold Helms, Paul Rissen et Jack Hayford. Au moment de son départ à la retraite en 1988, l'international Church comptait 10000 Eglises. Ce chiffre est passé, en 2004, à 30000 -

41. H. L. Mencken, A Mencken Chrestomathy, New York, Alfred A. Knopf, 1949, p. 292.

42. Jonathan Kay, «From Rural Ontario, a Preacher who Changed America », Free Republic, 25 juillet 2007, http://www.freerepublic.com/focus/freligion/1871554/posts (consulté en octobre 2008) 
soit plus de 4 millions de fidèles originaires de 123 pays $^{43}$. En 2003, l'International Church céda la station de radio KFSG-FM à la compagnie Spanish Broadcasting Systems pour la somme de 250 millions de dollars ${ }^{44}$. En 2006, Richard Rossi, acteur et producteur pentecôtiste très controversé, sortit un film intitulé Aimee Semple McPherson, retraçant la vie de la prédicatrice. Alors que des revues évangéliques comme Christianity Today, Charisma et Ministries Today lui réservèrent un accueil plutôt favorable, l'International Church of the Foursquare Gospel fit savoir qu'elle ne cautionnerait pas ce film ${ }^{45}$. C'est dire que plus de soixante ans après sa mort, Aimee $\mathrm{S}$. McPherson continue à faire débat ${ }^{46}$.

78 rue Henri Durre

59590 RAISMES

mokhtar.benbarka@univ-valenciennes.fr

43. Don Lattin, « Popular evangelist elected to head Foursquare Church », San Francisco Chronicle, 5 juin 2005, http://www.sfgate.com/cgi-bin/article.cgi?f=/c/ a/2004/06/05/BAGCO719211.DTL (consulté en octobre 2008)

44. Ibid.

45. http://www.answers.com/topic/international-church-of-the-foursquaregospel (consulté en octobre 2008)

46. Merci à M. Laurent Amiotte-Suchet (Observatoire des religions en Suisse, Université de Lausanne) pour ses précieuses remarques. 


\section{BIBLIOGRAPHIE}

BAHR, Robert, Least of All Saints, Englewood Cliffs, Prentice Hall, 1979.

Blumhofer, Edith L., Aimee Semple McPherson. Everybody's Sister, Grand Rapids, Michigan, William B. Eerdmans Publishing Company, 1993.

Chouleur, Jacques, «Le panorama religieux de l'Amérique des années 1920 », in Serge Ricard, The Twenties, Aix-en-Provence, Publications de l'Université de Provence, 1982, pp. 105-118.

Epstein, Daniel Mark, Sister Aimee. The Life of Aimee Semple McPherson, New York, Harcourt Brace Jovanovich Publishers, 1993.

Fecher, Charles, Mencken, New York, Alfred A. Knopf, 1978.

KAsPI, André, Les Etats-Unis au temps de la prospérité : 1919-1929, Hachette, 1994.

KAY, Jonathan, «From Rural Ontario, a Preacher who Changed America », Free Republic, 25 juillet 2007.

Lately, Thomas, Storming Heaven. The Lives and Turmoils of Minnie Kennedy and Aimee Semple McPherson, New York, William Morrow and Company, Inc., 1970.

LatTin, Don, « Popular evangelist elected to head Foursquare Church », San Francisco Chronicle, 5 juin 2005,

Mencken H. L., A Mencken Chrestomathy, New York, Alfred A. Knopf, 1949.

Semple McPherson, Aimee, This is That, Los Angeles, Foursquare Publications, 1923.

Semple McPherson, Aimee, The Story of My Life, Waco, World Books, 1951.

SheAfer, Silvia Anne, Aimee Semple McPherson, Philadelphia, Chelsea House Publishers, 2004.

\section{SITE INTERNET}

http://www.answers.com/topic/international-church-of-the-foursquaregospel

http://www.freerepublic.com/focus/f-religion/1871554/posts

http://www.sfgate.com/cgi-bin/article.cgi?f=/c/a/2004/06/05/BAGCO 719211.DTL 